\title{
Análisis Multi-Wavelet para la detección de conductividad ventricular anormal en señales ECG de alta resolución.
}

\author{
Artículo Original
}

Marco A. Reyna-Carranza', Raimon Jané-Campos².

${ }^{1}$ Instituto de Ingeniería, Campus Mexicali, Universidad Autónoma de Baja California (U.A.B.C.), Mexicali, B.C., México, ${ }^{2}$ Departamento de Ingeniería de Sistemas, Automática e Informática Industrial. Centro de Investigación en Ingeniería Biomédica (CREB). Universidad Politécnica de Cataluña, Barcelona, España.

\section{RESUMEN.}

Introducción. Los potenciales tardíos ventriculares (por sus siglas en inglés se abrevia VLP) son indicadores de conductividad ventricular anormal que se asocia a los substratos arrítmicos cardiacos en pacientes que han sobrevivido a un infarto de miocardio. Muchos investigadores han desarrollado distintos procedimientos para detectar anormalidades de conducción ventricular en señales electrocardiográficas (ECG) de alta resolución (por sus siglas en inglés se abrevia HRECG), mediante el uso de la transformada "wavelet". Sin embargo, muy pocos han logrado obtener resultados cuantitativos satisfactorios.

Materiales y Métodos. Aquí se propone analizar las señales HRECG en pacientes postinfartados, cuantificando el número de singularidades que presenta cada señal dentro del complejo QRS. Para la detección se utilizan varias funciones wavelets y no sólo una, como lo propone el método de Rubel y col. Los métodos se probaron con una base de datos compuesta por 132 registros HRECG. 59 registros HRECG fueron tomados de pacientes post-infartados y los 73 restantes se obtuvieron de sujetos sanos.

Resultados. Al usar más de una función wavelet en el análisis, se nota una substancial reducción en el número de falsos negativos (FN) (i.e., de 5 a 1 casos), sin que el número de falsos positivos (FP) se incremente considerablemente (i.e., de 9 a 11 casos). Por lo tanto, los valores predictivos del método se mejoran.

Discusión. Los VLP son formas de onda que cambian tanto en amplitud, forma y frecuencia,

Solicitud de sobretiros: Dr. Marco A. Reyna-Carranza, Depto. de Investigación, Dirección General de Investigación y Posgrado, Universidad Autónoma de Baja California, Edificio de Rectoría S/N, C.P. 21180, Mexicali, B.C., México.

Tel. (6) 566-36-33 Fax: (6) 566-09-15 E-mail: reyna@info.rec.uabc.mx

Recibido el 22/Mayo/2000. Aceptado para publicación el 22/Noviembre/2000.

Este artículo está disponible en http://www.uady.mx/ biomedic/rb011223.pdf

Vol. 12/No. 2/Abril-Junio, 2001 


\section{MA Reyna-Carranza, R Jané-Campos.}

como en localización temporal, debido a las características intrínsecas de los substratos arrítmicos. Una sola función wavelet no se puede ajustar a todas las estructuras inestables de cada HRECG de la base de datos. Por lo tanto, se sugiere el uso de un grupo de 4 funciones wavelet para resolver el problema. Si una de las cuatro wavelets no detecta la anormalidad de conducción, (debido a que la forma de onda no se ajusta al patrón de anormalidad de los VLP), aún es probable que alguna de las 3 wavelets restantes sí se ajuste y, por lo tanto, la singularidad sea detectada.

(Rev Biomed 2001; 12:98-110)

Palabras Clave: Potenciales-Tardíos, electrocardiograma de alta resolución, Wavelets, Diagnóstico no invasor.

\section{SUMMARY.}

Multi-Wavelet analysis for detecting abnormal ventricular conductivity in the high resolution ECG.

Introduction. Ventricular late potentials can be used for indicating abnormal ventricular conduction, because they are associated with the arrhythmogenic ventricular activity produced by damaged cardiac tissue after an infarct. Most researchers have designed procedures for detecting ventricular conduction abnormalities analyzing High Resolution ECG (HRECG) by means of the wavelet transform. However, few scientists have obtained satisfactory quantitative results.

Material and Methods. We are proposing to analyze HRECG's of post-infarcted patients by quantifying the singularities within the QRS complex. For their detection we use four wavelets, instead of one as the majority of the reported methods. We have tested the methods with a database of 132 HRECG recordings. 59 HRECG recordings were taken from postinfarcted patients, and the remaining 73 from healthy subjects.

Results. When using more than one wavelet in the analysis, the number of false negatives (FN's) was reduced from 5 to 1 , without considerably increasing the number of false positives (FP's) from 9 to 11 . Therefore, the predictive value of the method increased too.

Discussion. The abnormal ventricular potentials are unstable structures that not only change in amplitude, shape and frequency, but also in temporal localization, due to the intrinsic characteristics of the arrhythmic substrates. A single wavelet function can not adjust to the unstable structures for each HRECG in the database. Therefore, we are suggesting the use of a group of 4 wavelet functions to solve the problem. If one of the four wavelet functions does not detect the conduction abnormality (in case the shape can not be adjusted to the abnormality pattern), it is still possible that one of the remaining wavelets can be adjusted and, therefore, that the irregularity will be detected. (Rev Biomed 2001; 12:98-110)

Key words: Late-Potentials, high resolution electrocardiogram, Wavelets, Non-Invasive Methods.

\section{INTRODUCCIÓN.}

Los potenciales tardíos ventriculares (VLP) son componentes del electrocardiograma (ECG) de amplitudes muy pequeñas con un contenido frecuencial que se encuentra por arriba de los $40 \mathrm{~Hz}$, y se asocian a las enfermedades coronarias tales como infartos de miocardio y arritmias cardiacas (1). Se localizan comúnmente en la porción terminal del complejo QRS y/o al inicio del segmento ST, aunque a veces pueden también localizarse en cualquier parte dentro del complejo QRS (2).

Los VLP son muy utilizados como marcadores de riesgo arrítmico en el desarrollo de métodos no invasores para la predicción de

\section{Revista Biomédica}


muerte cardiaca súbita. Desafortunadamente, la mayoría de estos métodos presentan valores predictivos bajos, dado que los VLP son estructuras fisiológicas muy difíciles de detectar. Por ejemplo, el método del dominio temporal clásico (3) es efectivo únicamente cuando los VLP presentan una Relación Señal a Ruido (RSR) por arriba de 3, y además, su ubicación debe darse en la parte terminal del complejo QRS. Otros métodos como el espectro-temporal (4) permite RSR menores, pero está limitado al análisis del segmento ST, ya que presenta serios problemas espectrales al incluir en el análisis parte del complejo QRS.

La dificultad en la detección de los VLP estriba en que son estructuras que varían tanto en forma, amplitud, duración y ubicación sobre el trazo ECG. La figura 1 muestra varios VLP tomados de distintos pacientes que sobrevivieron a un infarto de miocardio.

Como puede verse, los VLP pueden variar en sus características estructurales, dependiendo de la antigüedad del infarto, volumen de tejido dañado por el infarto, localización del infarto, etc. Esto nos ha llevado a realizar algunas reflexiones en cuanto a los métodos de análisis que utiliza la transformada wavelet para detectar anormalidades de conducción ventricular, y que a continuación discutiremos. Se presentan algunos conceptos importantes de la teoría de wavelets para facilitar la comprensión de los razonamientos en el análisis de señales electrocardiográficas de alta resolución (HRECG) para detectar los VLP.

\section{Transformada Wavelet.}

Hace no más de 10 ó 15 años que la teoría de wavelets empezó a desarrollarse en el área del procesamiento de señales; primero Girard en 1982 y luego Morlet en 1983. No obstante, la teoría de wavelets está inspirada en las ideas que se habían originado durante los pasados 20 ó 30

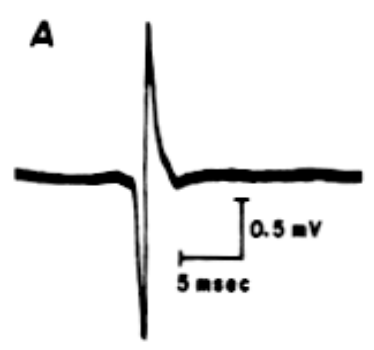

B $\quad C$

D
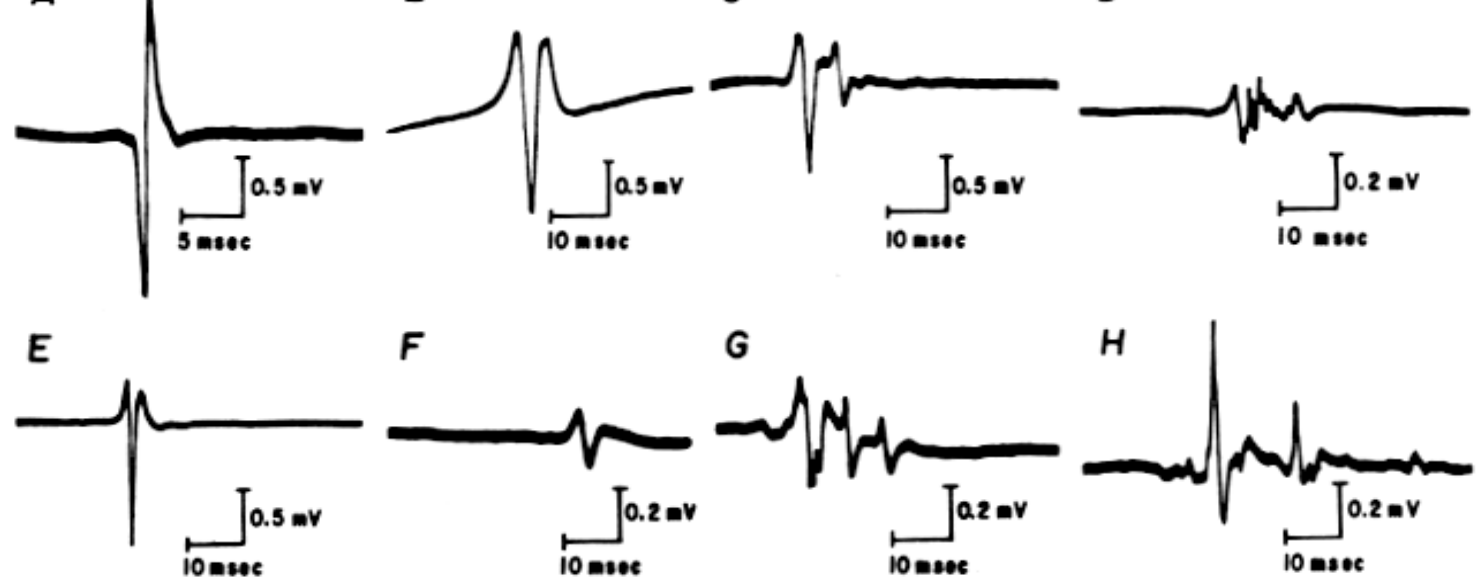

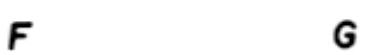

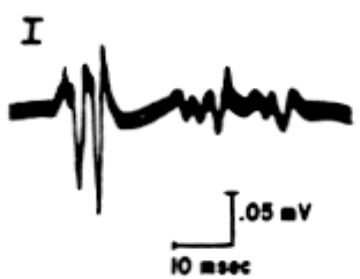

J
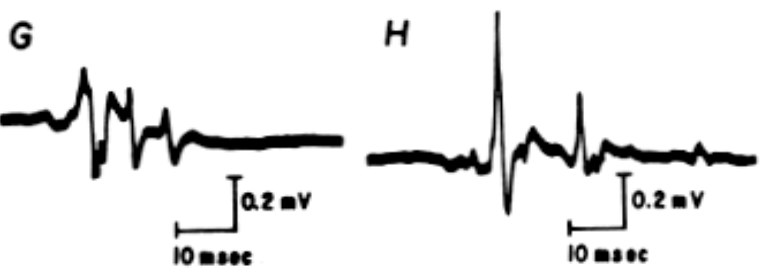

L

Figura 1.- VLPs de distintos pacientes. (a), (b) y (c) se registraron 5 días después de haber ocurrido el infarto, (d) y (g) se tomaron después de 2 semanas de ocurrido el infarto, (h), (k) y (l) son de 2 meses después de presentado el infarto, (e), (f), (i) y (j) son después de 6 meses de ocurrido el infarto. (De Garnder P, et al. Electrophysiologic and anatomic basis for fractionated electrograms recorded from healed myocardial infarcts. Circulation. 1985;72:596-611. (Figura tomada bajo permiso de la editorial Kluwer academic publisher. Ref: Gomes, Signal Averaged Electrocardiography, pp. 19 figure 6, 1993).

Vol. 12/No. 2/Abril-Junio, 2001 


\section{MA Reyna-Carranza, R Jané-Campos.}

años, principalmente en las áreas de ingeniería, física, y matemáticas puras (5). A partir de entonces se han venido desarrollando poderosas herramientas con las que se pueden representar y analizar señales de forma distinta a la tradicional con la transformada de Fourier. Es decir, ahora el análisis y descomposición de una señal puede hacerse por medio de escalas múltiples.

Para efectos prácticos, las wavelets se pueden separar en dos grupos: las wavelets redundantes o transformada wavelet continua (CWT), y las wavelets no redundantes o transformada walet discreta (DWT) (wavelets de bases ortogonales, semi-ortogonales, y biortogonales). Las wavelets redundantes producen mejores resultados en el análisis de señales, y en la detección y extracción de características, ya que brindan una completa descripción espectro-temporal de la señal analizada. Las wavelets no redundantes, dada la ortogonalidad, presentan resultados muy buenos cuando se necesita comprimir cierta cantidad de datos para su almacenamiento o transmisión, y/ o cuando la ortogonalidad de la representación se considera un factor importante para evitar la redundancia de información.

Dado que el interés de nuestro estudio es el análisis y la extracción de características de las señales HRECG, y no la compresión de datos, en este subapartado se revisará únicamente la teoría asociada a las wavelets redundantes, es decir la CWT.

Una forma de interpretar el análisis por wavelets es entendiendo que una función wavelet es una función base, de la misma forma que las exponenciales complejas son funciones base en el análisis de Fourier. La transformada de Fourier (FT):

$$
\operatorname{STFT}(t, \omega)=\frac{1}{\sqrt{2 \pi}} \int_{\infty} s(t) h(\tau-t) e^{-j \omega \tau} d \tau
$$

brinda información valiosa cuando la señal bajo análisis no presenta cambios drásticos en el tiempo, i.e., si la señal es estacionaria. Sin embargo, muchas señales presentan características no-estacionarias, es decir, características transitorias tales como: discontinuidades, cambios abruptos, componentes transitorios, etc. Estas características de la señal suelen ser las más importantes al hacer el análisis, y la transformada de Fourier se ve imposibilitada para detectarlas.

En 1946 Dennis Gabor realizó una adaptación a la transformada de Fourier para tratar de superar esa deficiencia. La nueva adaptación consistió en analizar la señal por secciones. El proceso se lleva a cabo mediante la multiplicación de una ventana con la señal. Gabor le llamó a esta técnica Short-Time Fourier Transform, (STFT) (5); la cual convierte una señal del dominio temporal, en otra señal bidimensional en tiempo y frecuencia:

$$
\operatorname{STFT}(t, \omega)=\frac{1}{\sqrt{2 \pi}} \int_{\infty} s(t) h(\tau-t) e^{-j \omega \tau} d \tau
$$

en donde : $h(t)$ es la ventana y $s(t)$ es la señal.

Desafortunadamente, esta transformación no resuelve del todo las limitaciones que la FT presenta, ya que su resolución en tiempofrecuencia se asocia a un compromiso de precisión en cuanto a la localización de transitorios. El compromiso de resolución, ya sea en tiempo o frecuencia, dependerá de la longitud de la ventana que se use. La limitación que presenta la $S T F T$ en cuanto a la rigidez de la longitud de la ventana, queda resuelto con la transformada wavelet mediante la dilatación y contracción de sus funciones base en forma automática dependiendo de la variación de la frecuencia. Básicamente, en el análisis por wavelets, la selección de la ventana de la señal analizada se realiza en regiones de tamaño variable. Esta técnica permite una mayor precisión en frecuencia a bajas frecuencias, mediante la dilatación de las ventanas en el tiempo, y también una mayor precisión temporal a altas frecuencias mediante la contracción de las ventanas en el tiempo.

\section{Revista Biomédica}




\section{Análisis Multi-Wavelet en ECG de alta resolución.}

La $C W T$ se define matemáticamente de la siguiente forma:

$$
C W T(b, a)=\frac{1}{\sqrt{a}} \int_{\infty} \psi^{\prime}\left(\frac{t-b}{a}\right) s(t) d t .
$$

Esencialmente, la $C W T$ se usa para realizar un análisis de correlación por escalas, en donde el resultado de la transformación $C W T(b, a)$ será máximo cuando el parecido de la señal bajo análisis $s(t)$ con la función wavelet $\psi$ ' $\left(\frac{t-b}{a}\right)$ sea también máximo en la escala $a$. Por ejemplo, sea el modelo $f(x)=\varphi_{a}(x-\Delta x)+\eta(x)$, en donde $\varphi_{a}(x / a)$ es una señal determinística conocida a una escala $a, \Delta x$ un parámetro de localización desconocido y $\eta(x)$ una componente de ruido blanco gausiano aditivo. La teoría plantea que el procedimiento óptimo para estimar $\Delta x$ es mediante el cálculo de la correlación entre la función modelo $\varphi_{a}(x / a)$, y la función a analizar $f(x)$ para cada posición $\Delta x$, y luego seleccionar la posición que corresponda al valor de correlación máximo. La mayoría de las veces no se tiene el conocimiento a priori del tamaño de escala de la señal que se quiere detectar. Por lo que se podría variar el factor de escala $a$ de la señal modelo para cada cálculo de correlación.

Con la misma idea se puede usar una función wavelet (o varias) como modelo de detección de ciertos patrones, por ejemplo, la detección de VLP en el análisis de señales electrocardiográficas (HRECG).

La teoría de wavelets es muy vasta y al lector interesado se le recomienda revisar el libro de Daubechies (6 ), además del excelente tutorial que han publicado Riol y Vetterli (7).

\section{Transformada Wavelet de Señales ECG de Alta Resolución.}

Quizá el único procedimiento de análisis por wavelets que ha brindado resultados cuantitativos es el de "detección de singularidades o irregularidades", propuesto por Rubel y col. (8). En el siguiente subapartado haremos una revisión de este procedimiento, al cual lo llamaremos análisis Uni-Wavelet, para distinguirlo del procedimiento que hemos propuesto y que será revisado en el subapartado A. 2

A. Procedimiento para la Detección de Singularidades

\section{A.1. Análisis Uni-Wavelet}

El procedimiento de este análisis (8), se basa en la detección de singularidades producidas por irregularidades de conducción cardiaca reflejadas a lo largo de la señal HRECG.

Para detectar las singularidades se efectuaron distintas pruebas de detección mediante la aplicación de funciones wavelet, obteniendo resultados predictivos muy variados. Las funciones wavelet que se probaron fueron:

1. Morlet.- Definida de la siguiente forma: $\psi(t)=\exp \left(i_{0} t\right) \exp \left(-t^{2} / 2\right)$

2. Primeras seis derivaciones de la función Gausiana definida como: $g(t)=\exp \left(-t^{2} / 2\right)$

3. Wavelets "B-spline" de grado $n$, definidas de la siguiente forma:

$$
g^{n}(n) \approx \sqrt{\left(\frac{6}{\pi(n+1)}\right)} \exp \left(\frac{-6 t^{2}}{(n+1)}\right)
$$

4. Meyer.- Se define en el dominio de la frecuencia de la siguiente forma: $\psi(f)=\exp (-j)$ $\theta(f)$ siendo $\psi(t)=F T^{-1}(\Psi(f))$, en donde:

$$
\begin{array}{ll}
\theta(f)=\theta(-f) & \forall f \\
\theta(f) \neq 0 & \forall|f| \in[1 / 2-\varepsilon, 1+2 \varepsilon] \\
\theta(f)=1 & \forall|f| \in[1 / 2+\varepsilon, 1-2 \varepsilon] \\
\theta^{2}(f)+\theta^{2}(1-f)=1 & \forall|f| \in[1 / 2-\varepsilon, 1+2 \varepsilon] \\
\theta(2 f)+\theta(1-f)=1 & \forall|f| \in[1 / 2-\varepsilon, 1+2 \varepsilon] \\
\text { con } 0<\varepsilon \leq 1 / 6 . &
\end{array}
$$

Por último se obtiene la función wavelet $(t)$ mediante el cálculo de la transformada inversa de Fourier:

$$
\psi(t)=F T^{-1}(\psi(f))
$$

a). Vector Magnitud de la Transformada Wavelet. 


\section{MA Reyna-Carranza, R Jané-Campos.}

Como se mencionó, el método se basa en la detección de singularidades producidas por irregularidades de conducción cardiaca reflejadas a lo largo del complejo QRS de la señal HRECG. Para ello se obtiene primeramente el vector magnitud de la transformada wavelet para cada una de las derivaciones ECG ortogonales $X, Y$ y $\mathrm{Z}$, de la siguiente manera:

$$
W T M(a, n)=\sqrt{W T X^{2}(a, n)+W T Y^{2}(a, n)+W T Z^{2}(a, n)}
$$

En donde $W T X$ es la magnitud de la transformada wavelet de la derivación $X, W T Y$ es la magnitud de la transformada wavelet de la derivación $Y, W T Z$ es la magnitud de la transformada wavelet de la derivación $Z$ y, WTM es el vector magnitud de la transformada wavelet.

De esta forma, por cada señal se obtendrán tres representaciones en tiempo-escala. De estas, se obtendrá otra representación en tiempo-escala sintetizando la información contenida dentro de cada una de las derivaciones ortogonales.

El factor de escala $a$ se determina como $a$ $=2^{-m}$, en donde $m$ varía linealmente. El máximo valor de $m$ se establece de tal forma que la frecuencia central de la wavelet de máxima escala no esté por debajo de $25 \mathrm{~Hz}$, y para que la wavelet de mínima escala no esté por arriba de $250 \mathrm{~Hz}$. Así, la banda de frecuencia de análisis queda establecida entre los 25 y $250 \mathrm{~Hz}$ (7 escalas para siete valores de $m$ ), lo cual cubriría la banda frecuencial para la que se intuye que se encuentran los VLP en pacientes con riesgo de arritmias.

\section{b). Detección de las Singularidades.}

La detección de las singularidades o estructuras irregulares se realiza sobre cada escalamiento en la dirección temporal. Esto consiste en precisar temporalmente la evolución de las singularidades mediante máximos locales para cada escalamiento realizado por la transformada wavelet en curso. El método se compone de tres pasos:

\section{Revista Biomédica}

1. Detección de los máximos locales.- Se detectan los máximos locales para cada una de las escalas. El método define un máximo local como el punto de amplitud máxima entre la pendiente de subida y la pendiente de bajada de una cresta. Se consideran como máximos locales válidos, aquellos que tienen una magnitud mayor que un umbral de amplitud establecido (AMPLTHR) (ver figura 2(a)). Esto se hace con el fin de descartar máximos locales de muy

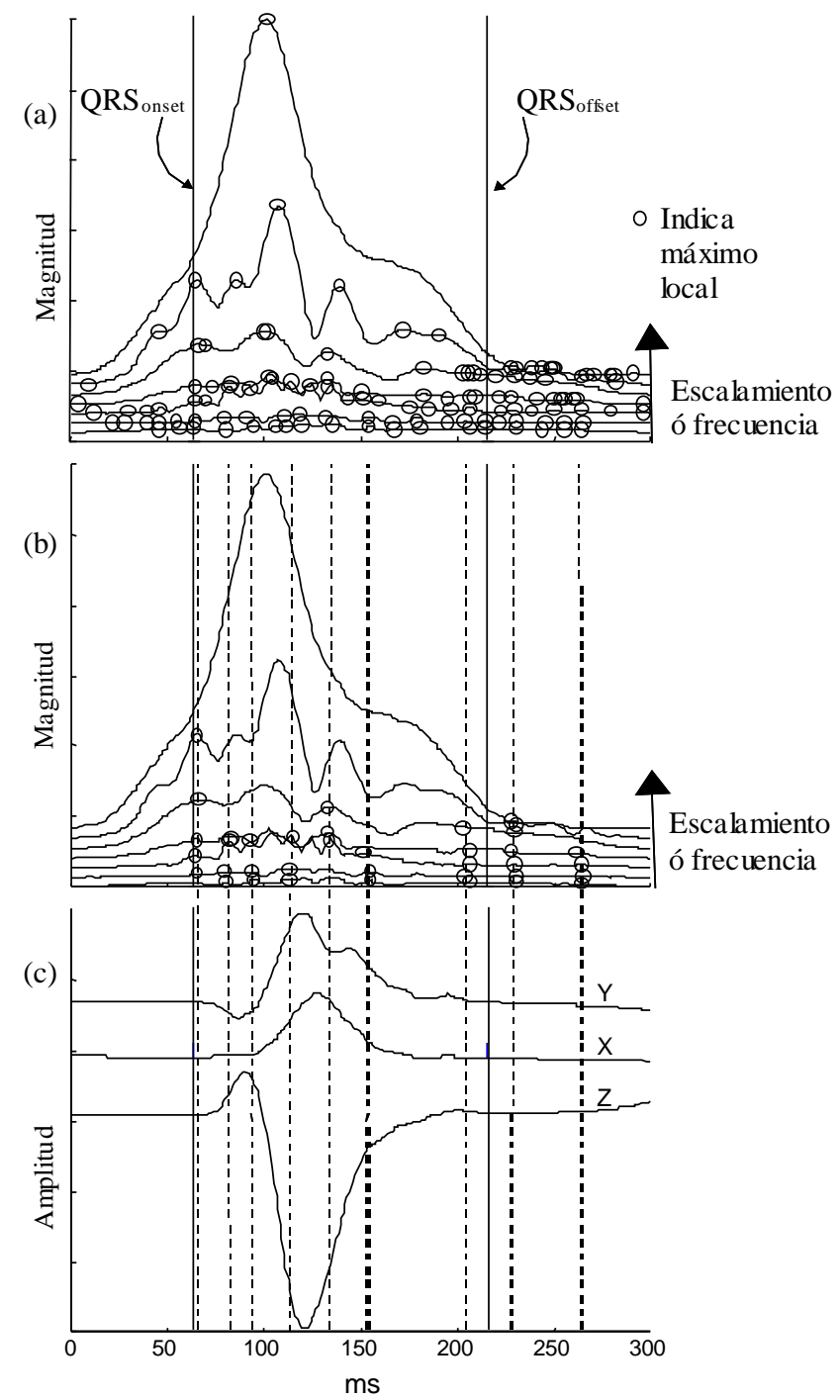

Figura 2.- Detección y localización de singularidades en señales HRECG. (a) Máximos locales sobre cada WTM. (b) Encadenamiento de los máximos locales (líneas punteadas). (c) Singularidades proyectadas sobre los trazos de HRECG (líneas punteadas). Las líneas continuas verticales señalan el inicio (QRSonset) y final del QRS (QRSoffset) respectivamente. 


\section{Análisis Multi-Wavelet en ECG de alta resolución.}

pequeñas amplitudes debidos al ruido. El umbral se determina como un porcentaje de la amplitud máxima de la transformada wavelet a la mínima escala.

2. Localización de la conectividad de los máximos locales.- Se dice que un máximo local de cualquier escala tiene conectividad con un máximo local de la mínima escala si la desviación entre sus respectivas localidades temporales no es mayor de un umbral de dispersión (SCATTHR).

3. Aceptación de un máximo local como singularidad.- La singularidad se detecta cuando el número de máximos locales con conectividad es mayor que un número previamente establecido, al cual se le llama RIDGLEN (número mínimo de crestas interconectadas). Para el ejemplo que se muestra en la figura 2(b) el número mínimo de crestas interconectadas es de 3 .

La asignación de los valores para los parámetros AMPLTHR, SCATTHR y RIDGLEN define la detección de las singularidades sobre las señales HRECG, y los resultados serán diferentes para cada asignación sobre el segmento de señal que se analice. El método considera un segmento de señal definido por $\left[\mathrm{QRS}{ }_{\text {onset }}, \mathrm{QRS}_{\text {onset }}+250 \mathrm{~ms}\right.$ ], en donde $\mathrm{QRS}_{\text {onset }}$ se refiere al inicio del complejo QRS.

\section{A.2. Análisis Multi-Wavelet}

La CWT es un proceso que mide el grado de correlación entre el segmento de señal en análisis y la función wavelet con la escala en curso. Este mismo principio se aplica en el análisis multi-wavelet el cual se explicará en este subapartado, pero antes analicemos el siguiente ejemplo de simulación. Sea una señal HRECG real con una duración del QRS (QRSd) igual a $163 \mathrm{~ms}$. La señal ha sido filtrada en paso-bajo en una banda de $0-50 \mathrm{~Hz}$ con el objeto de sumarle señales componentes, los cuales simularán tanto potenciales de alta frecuencia $x_{1}(t)$ como los VLPs $x_{2}(t)$ (ver figura 3(a-e)). También se sumará una componente de ruido blanco gausiano $n(t)$. Es decir, el modelo construido se puede representar mediante la siguiente relación:

$H R E C G_{\text {sim }}(t)=H R E C G_{\text {filt }}(t)+x_{1}(t)+x_{2}(t)+n(t)$, [1]

en donde $H R E C G_{\text {filt }}(t)$ es la señal ECG de alta resolución de la base de datos, pero filtrada, y $H R E C G_{\text {sim }}(t)$ es la señal HRECG simulada. $x_{1}(t)$ $=a \operatorname{sen}\left(2 \pi f_{0} t\right) \exp \left((t-125 \mathrm{~ms})^{2} / 2 b\right)$, donde $a$ es igual al $12 \%$ de la amplitud máxima de la señal ECG, y $f_{0}=100 \mathrm{~Hz}$. El factor de escala $b$
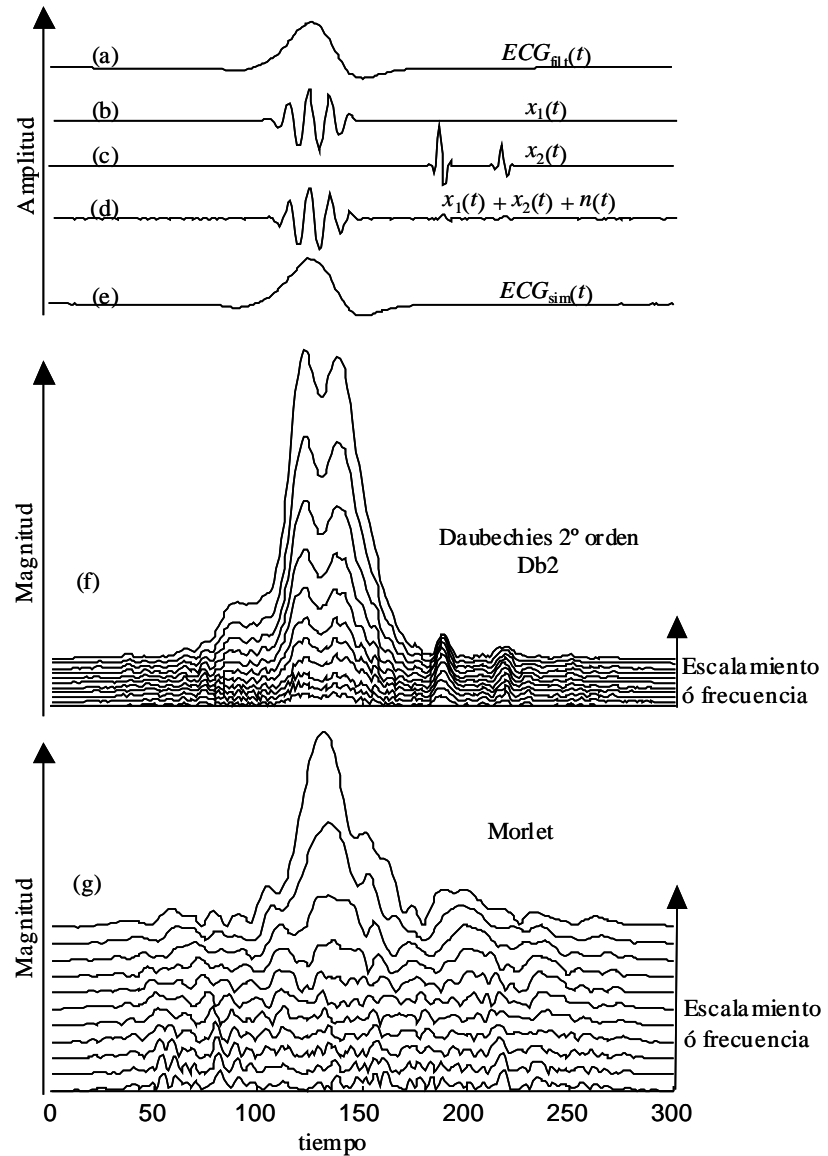

Figura 3.- Dos representaciones tiempo-escala para detectar VLP simulados. (a) HRECG filtrada en paso-bajo 0-40 Hz. (b) Potenciales QRS por arriba de $40 \mathrm{~Hz}$. (c) VLP simulados. (d) Suma de los VLP con los potenciales de alta frecuencia y con ruido blanco gausiano aditivo. (e) HRECG que se compone de la sumatoria de todas las señales anteriores. (f) Representación tiempo-escala de la señal (e) mediante la wavelet Db2. (f) Representación tiempo-escala de la señal (e) mediante la wavelet Morlet.

Nótese la clara localización de los VLP simulados cuando se aplica la wavelet Db2, y la pésima localización de los VLP cuando se emplea la wavelet de Morlet.

Vol. 12/No. 2/Abril-Junio, 2001 
MA Reyna-Carranza, R Jané-Campos.

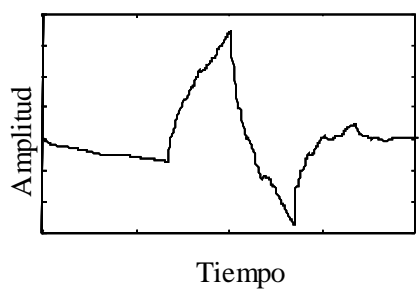

(a)

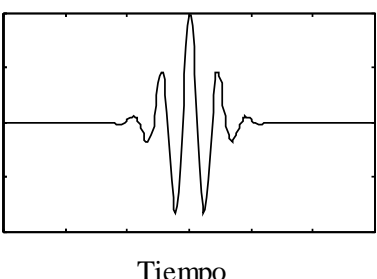

(b)
Figura 4.- (a) Función wavelet Daubechies de $2^{\circ}$ orden. (b) Función wavelet Morlet.

se hace igual a 20 para dar a la señal una duración efectiva (soporte compacto) de 50ms. $x_{2}(t)=c$ $\operatorname{sen}\left(2 \pi f_{1} t\right) \exp \left((t-180 \mathrm{~ms})^{2} / 2 d\right)+c / 2 \operatorname{seno}(2$ $\left.\pi f_{0} t\right) \exp \left((t-218 \mathrm{~ms})^{2} / 2 d\right)$, donde c es el $2 \%$ de la amplitud máxima de la señal ECG, y el factor de escala $d$ se hace igual a 3, para dar a cada patrón VLP simulado una duración efectiva de $18 \mathrm{~ms}, f_{1}=150 \mathrm{~Hz}$.

La figura 3(f) es la representación tiempoescala de la HRECGsim (figura 3(e)) que se obtiene mediante la aplicación de la CWT usando la función wavelet de Daubechies de $2^{\circ}$ orden (Db2). La figura 3(g) es la representación tiempo-escala de la misma HRECGsim que se obtiene también mediante la CWT, pero ahora empleando la función wavelet Morlet. Viendo ambas representaciones tiempo-escala, se nota que la que se obtuvo con el empleo de la wavelet Db2 (figura 4(a)) es la que mejor detecta los potenciales anormales simulados, dado que su parecido con estos, es mucho mayor que el parecido que se presentó con la función wavelet de Morlet (ver figura 4(b)).

Ahora, supóngase que la HRECGsim de la figura 5(e) es una señal ECG distinta a la de la figura 3(e), pero que responde al mismo modelo de la relación [1]. En la figura 5(d) se pueden ver los nuevos VLPs. Como puede observarse, la forma de estos potenciales es completamente distinta a los de la HRECGsim anterior (figura 3). Su parecido es ahora mayor para la wavelet Morlet que para la wavelet Db2. Por lo tanto, se esperaría obtener mejores resultados de detección de los VLPs con la wavelet de Morlet que con la wavelet de Db2.
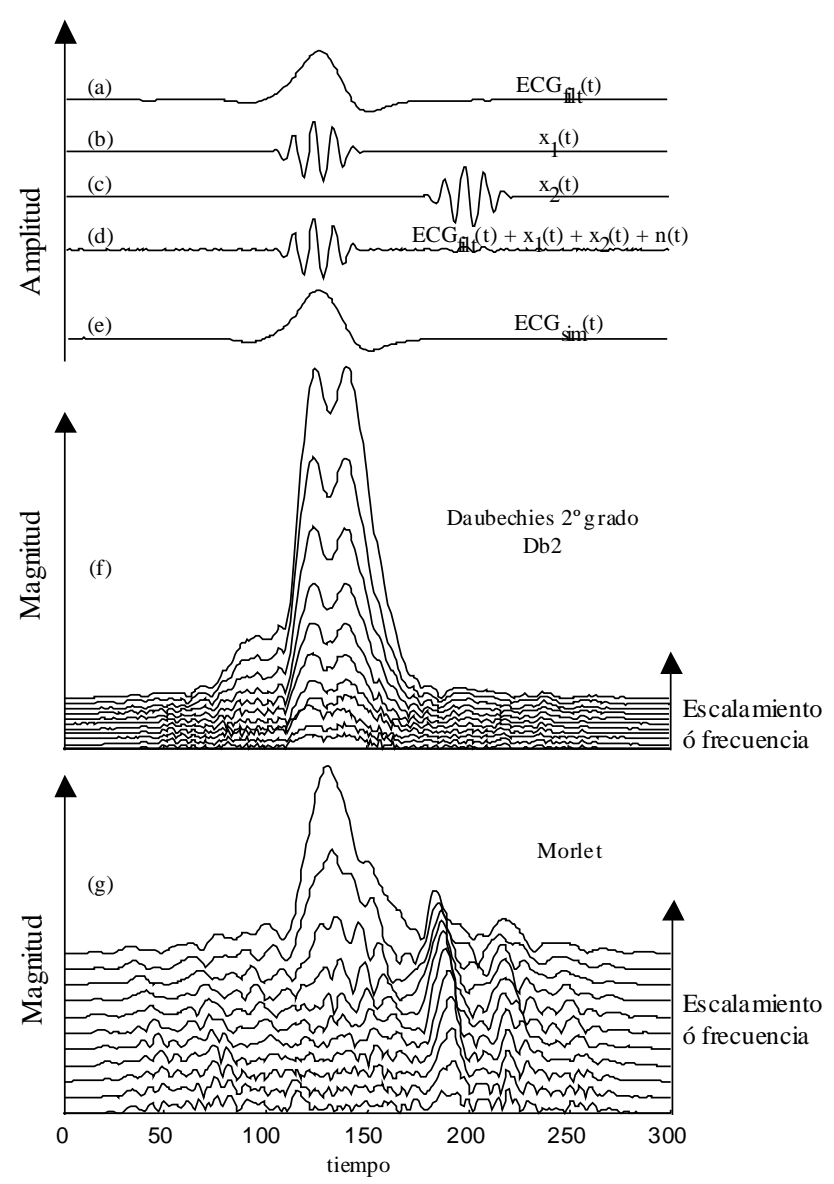

Figura 5.- (a-b) Mismas señales de la figura 3, (c) VLP simulados con distintinta morfología que los simulados en el caso de la figura 3(c), (d) es la sumatoria de las señales (b y c) más una componente de ruido blanco gausiano. $\mathrm{Al}$ obtener la representación tiempo-escala de la señal (e) aplicando la wavelet Db2, se puede apreciar que la localización de los VLP es prácticamente nula, sin embargo, al obtener la representación tiempo-escala de la misma señal (e) empleando la wavelet de Morlet, la localización de los VLP es muy clara. Debe notarse, que este caso es contrario al de la figura 3, ya que ahora la wavelet de Morlet es la que se ajusta mejor a la forma de los VLP simulados, por lo que genera mejores resultados que la wavelet $\mathrm{Db} 2$.

Si se observan las gráficas tiempo-escala (fig. $5 \mathrm{f}-\mathrm{g}$ ) respectivas a cada CWT (i.e., Db2 y Morlet), se corroborará la anterior aseveración.

Como se recordará, el método uniwavelet selecciona una sola función wavelet para el análisis de las HRECG, pero aunque se seleccione la que mejores resultados predictivos arroje para toda la población analizada, ésta no

\section{Revista Biomédica}




\section{Análisis Multi-Wavelet en ECG de alta resolución.}

podrá ser la mejor para detectar los potenciales ventriculares anormales de cada una de las diferentes HRECG en particular. Es decir, los resultados predictivos que se obtienen, son una medida del análisis global de todas las señales HRECG analizadas, ya que los resultados dependen única y exclusivamente del parecido de los VLPs de cada señal con la función wavelet seleccionada. Como los VLPs son micropotenciales de forma y posición impredecible, debido a la naturaleza del tejido cardiaco dañado por el infarto, una única forma de wavelet, no podrá detectar todas las formas posibles que se pudieran presentar en las HRECG de cada paciente en particular.

Dado lo anterior, se propone mejorar el método uni-wavelet mediante un procedimiento que aumenta su capacidad para detectar un mayor número de pacientes con riesgo de taquicardia ventricular. La innovación del método, como se verá en los resultados del análisis de la base de datos, incrementa ligeramente el número de casos FPs, pero el número de casos FNs lo disminuye considerablemente.

Algoritmo.

El algoritmo que hemos propuesto, consiste en analizar cada HRECG de la misma base de datos, con 4 funciones wavelets distintas. En caso de que alguna de las wavelets detecte singularidades considerables sobre la señal HRECG en curso, esta será considerada como de riesgo para desarrollar taquicardia ventricular. Las wavelets usadas se escogieron

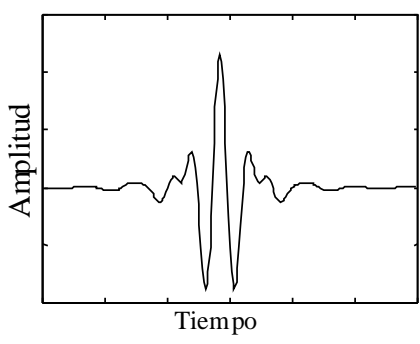

(a)

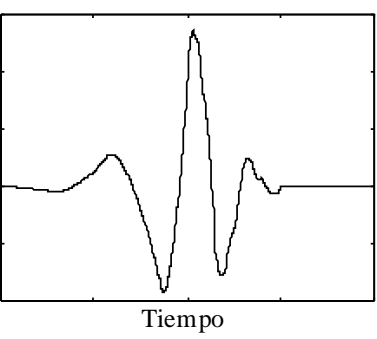

(b)
Figura 6.- (a) Función wavelet Meyer y (b) función wavelet Daubechies de $4^{\circ}$ orden. con base al parecido con la morfología de los potenciales anormales que pudieran ser más comunes, según estudios patofisiológicos sobre VLPs realizados en post-infartos crónicos (9).

Dado que ni la morfología ni las amplitudes de los VLPs son los mismos para cada una de las señales HRECG de cada paciente, el papel de las wavelets seleccionadas es detectar estas variaciones de los VLPs.

Las 4 wavelets utilizadas en el análisis son las siguientes:

1. Morlet. Se ha definido en el subapartado III.A.1.

2.Meyer. Definida también en el mismo subapartado.

3. Daubechies. (Daubechies de $2^{\circ}$ orden (Db2) y de $4^{\circ}$ orden $\left.(\mathrm{Db} 4)\right)$. No tiene expresión explícita. No obstante, esta se puede determinar con el módulo al cuadrado de su función de transferencia $h$, de la siguiente forma:

Sea $P(y)=\sum_{k=0}^{N-1} C_{k}^{N-1+k} y^{k}, \quad$ donde $C_{k}^{N-1+k}$

denota los coeficientes de Daubechies.

Luego:

$$
\left|m_{0}\left(\omega_{0}\right)\right|^{2}=\left(\cos ^{2} \frac{\omega}{2}\right)^{2} p\left(\operatorname{sen}^{2} \frac{\omega}{2}\right)
$$

Donde:

$$
m_{0}(\omega)=\frac{1}{\sqrt{2}} \sum_{k=0}^{2} h_{k} e^{-i k \omega}
$$

El soporte de esta wavelet está dada por $2 N-1 . N$ determina el orden de la wavelet (17). La figura 6 nuestra las formas para las wavelets de Meyer y de Daubechies de $4^{\circ}$ orden.

La figura 7 muestra el diagrama de flujo que describe el análisis utilizado para la detección de las singularidades de las señales HRECG mediante la CWT usando 4 distintas funciones wavelet. Cada señal HRECG es analizada primeramente por la wavelet Morlet, si ésta detecta que el número de irregularidades es igual o mayor que el umbral establecido, entonces se marca como señal con riesgo

Vol. 12/No. 2/Abril-Junio, 2001 
MA Reyna-Carranza, R Jané-Campos.

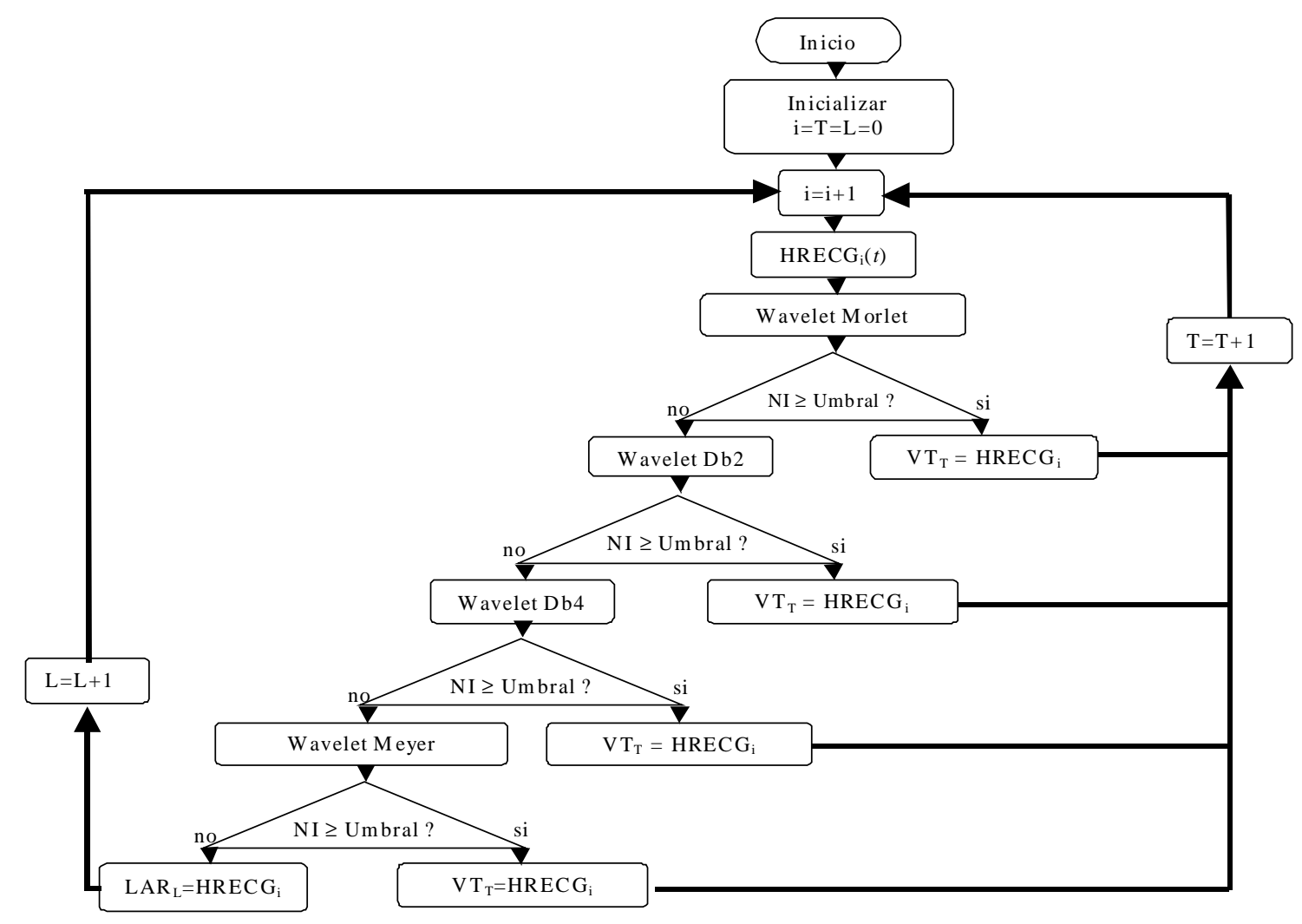

Figura 7.- Diagrama de flujo para ejecutar el análisis multi-wavelet.

arrítmico y se toma la siguiente señal HRECG de la base de datos. En caso de que el número de irregularidades detectado sea menor que el umbral, entonces la misma señal HRECG se analiza con la siguiente función wavelet. $\mathrm{Si}$ después de haber analizado la misma señal HRECG con las 4 distintas funciones wavelet, no se encontró el número mínimo de irregularidades para ser considerada con riesgo arrítmico, entonces el proceso la marca como señal HRECG de bajo riesgo de arritmia, y se continua con la siguiente señal HRECG de la base de datos. Las irregularidades se determinan con el mismo procedimiento usado en el método uni-wavelet. Los umbrales de los parámetros AMPLTHR, SCATTHR y RIDGLEN para las 4 distintas funciones wavelet se establecen con los mismos valores que se usaron en el análisis uni-wavelet empleando el tipo Morlet. Ello nos permitirá realizar comparaciones entre los resultados de Revista Biomédica aquel caso y el actual.

\section{SEÑALES HRECG ESTUDIADAS.}

a). Población de Estudio.

Grupo VT (infarto de miocardio con riesgo de taquicardia ventricular). Consiste de 59 señales HRECG de sujetos post-infartados. Estos individuos fueron internados en el Veterans Affairs Medical Center en Oklahoma City, en donde se les realizó el estudio electrofisiológico después de sobrevenido el infarto de miocardio.

Grupo LAR (sin infarto de miocardio, bajo riesgo de taquicardia ventricular). Consiste de 73 señales HRECG tomadas de sujetos a quienes no se les diagnosticó ningún signo de infarto de miocardio previo. El estudio electrofisiológico fue también realizado en el Veterans Medical Center en Oklahoma City.

b). Adquisición de la Población de Estudio. 
Se adquirieron registros ECG ortogonales bipolares de alta resolución (16 bits) de las derivaciones $\mathrm{X}$, Y y Z. Las derivaciones fueron adquiridas mediante el sistema PREDICTOR SAECG de la casa Corazonix Corp. en Oklahoma City. Se utilizó una frecuencia de muestreo de $2 \mathrm{KHz}$. Promediando la señal se logró situar el nivel de ruido por debajo de $0.4 \mathrm{~V}$ en valor RMS para los dos grupos. La medición del ruido se efectuó sobre el segmento ST del vector magnitud filtrado en pasa-altas a $40 \mathrm{~Hz}$.

\section{RESULTADOS.}

a). Análisis uni-wavelet

Se analizaron las 132 señales HRECG de ambos grupos. El AMPLTHR se estableció en 4\%, el SCATTHR fue de $3 \mathrm{~ms}$, la RIDGLEN fue igual a 3 y el segmento de señal ECG que se analizó quedó establecido iniciando en QRSonset y finalizando en QRSonset $+\mathrm{T}$, en donde $\mathrm{T}=$ $114 \mathrm{~ms}+80 \mathrm{~ms}$. El inicio del QRS (QRSonset) fue detectado mediante el sistema PREDICTOR SAECG. La media del Número de Irregularidades (NI) y los valores predictivos del análisis se muestra en el cuadro 1 (a). La población de estudio empleada para probar el método de análisis multiwavelet fue la misma que se usó en el método uni-wavelet.

En el cuadro 1 (b) se dan los valores predictivos que se obtuvieron al analizar la base de datos completa con las 4 funciones wavelet. Con el objeto de normalizar criterios de comparación, el NI umbral se estableció igual al caso del análisis con una sola función wavelet (uni-wavelet).

El analizar la base de datos con distintas wavelets, reduce el número de casos FNs (i.e., de 5 a 1), sin aumentar demasiado el número de casos FPs (i.e., de 9 a 11). La media de los valores NI para cada método se muestran también en la tabla I(b).

\section{DISCUSIÓN Y CONCLUSIONES.}

Los potenciales anormales de conducción ventricular son estructuras que cambian tanto en forma como en localización temporal y de frecuencia, debido a los substratos arrítmicos en el tejido cardiaco dañado por el infarto. Una

\section{Cuadro 1}

(a) Resultados predictivos del procedimiento original mediante la wavelet Morlet (uni-wavelet), (b) resultados predictivos mediante el análisis multi-wavelet.

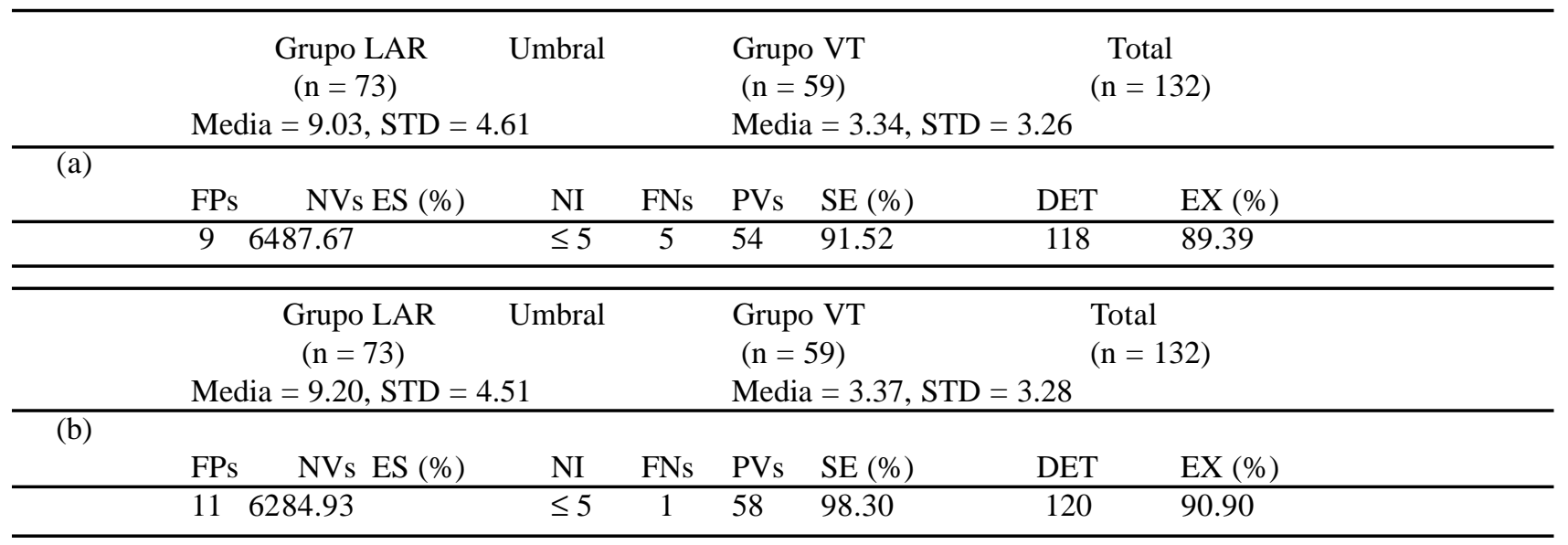

$L A R=$ grupo de bajo riesgo arrítmico; $V T=$ grupo de alto riesgo arrítmico. FPs = falsos positivos; $N V s=$ negativos verdaderos; $E S=$ porcentaje de casos no-patológicos correctamente clasificados; FNs = falsos negativos; $P V s=$ positivos verdaderos; $S E=$ porcentaje de casos patológicos correctamente clasificados; DET = pacientes detectados; $E X=$ porcentaje total de casos correctamente clasificados; $N I=$ umbral de números de irregularidades; Total = total de pacientes analizados. Media = valor medio del NI; STD = desviación estándar del NI.

Vol. 12/No. 2/Abril-Junio, 2001 


\section{MA Reyna-Carranza, R Jané-Campos.}

sola función wavelet no podría ajustarse a estas estructuras para cada una de las señales HRECG. Por lo tanto, se sugiere emplear un grupo de funciones wavelet para resolver el problema. Si una de las wavelets no detecta la anormalidad de conducción, dado que su forma no se ajusta a la del patrón de anormalidad, es más probable que alguna de las otras funciones wavelet sí se ajuste y por lo tanto detecte la irregularidad.

Los valores predictivos que se obtuvieron con la innovación del método mejoraron considerablemente. El número de FNs disminuyó notablemente y el aumento del número de FPs fue muy leve.

Con lo anterior se demuestra, que con el empleo de una única función wavelet el análisis es menos eficiente en la detección de las irregularidades de conducción ventricular, ya que el principio de funcionamiento de la CWT, es medir el grado de similitud entre la función wavelet y el patrón de señal que se quiere localizar. El patrón de señal en este caso son los potenciales anormales de conducción ventricular.

Uno de los inconvenientes del análisis de HRECGs mediante la CWT, al igual que para todos los métodos que se proponen en la literatura, es su sensibilidad al ruido. Dado que el análisis que realiza la CWT es local, si la función wavelet se ajusta a algún patrón localizado de ruido, se generaría un coeficiente de correlación alto, interpretándose erróneamente como patrón de irregularidad ventricular. En nuestro caso de estudio estos errores se atribuyen a algunos de los casos FPs.

Otra limitación del método, es que no se han realizado las suficientes pruebas como para poder establecer un estándar en sus umbrales AMPLTHR, SCATTHR, RIDGLEN. Además, los umbrales podrían variar en función de la o las funciones wavelet que se apliquen.

En este trabajo de investigación se han analizado bases de datos de pacientes extranjeros, debido a que en nuestro país aun no se cuenta con este tipo de señales. Sin embargo, se está acondicionando un laboratorio de ingeniería biomédica en el Instituto de Ingeniería de la Universidad Autónoma de Baja California para generar bases de datos de señales HRECG.

Un análisis más exhaustivo de estos algoritmos constituye una extensión del trabajo de investigación, en donde se incluirán bases de datos con señales registradas de pacientes mexicanos.

\section{REFERENCIAS.}

1.- Breithardt G, Cain M, El-Sherif N, Flowers N, Hombach V, Janse M, et al. Standards for analysis of ventricular late potentials using high-resolution signalaveraged electrocardiography. A joint statement. Task Force committee of the European Society of Cardiology, the American Heart Association and the American College of Cardiology. J Am Coll Cardiol 1991; 17:9991006.

2.- Gomis PR. Análisis de AIQP en la predicción de eventos arrítmicos. Caracterización de potenciales anormales intra-QRS en el ECG de alta resolución: aplicación en la evaluación de cardiopatías [Tesis Doctoral]. Barcelona, Inst. de Cibernética, UPC.; 1996.

3.- Simson MB. Use of signals in the terminal QRS complex to identify patients with ventricular tachycardia after myocardial infarction. Circulation 1981; 64:235-42.

4.- Haberl R, Jilge G, Pulter R, Steinbigler P, Steinbeck G. Spectrotemporal mapping of the surface ECG for detection of ventricular late potentials. Signal Average Electrocardiography 1993; 125-36.

5.- D Gabor. Theory of communication. Journal of Institution of Electrical Engineers (London) 1946; 93:429-57.

6.- Daubechies. Ten Lectures on Wavelets, SIAM 1992.

7.- Riol O, Vetterli M. Wavelets and Signal Processing. IEEE Signal Processing Magazine 1991; 8 (4): 14-38.

8.- Rubel P, Courdec J Ph, Morlet D, Fayn J, Peyrin F,

\section{Revista Biomédica}


Touboul P. Spectral analisys of high-resolution ECGs. En: Moss A.J, and Stern S, editores. Noninvasive Electrocardiology. Clinical Aspects of Holter Monitoring. London: W. B. Saunders Company Ltd; 1996. p. 291-314.

9.- Mehra R. Pathophysilogy of late potentials: Experimental observation of late potentials. En: Gomes JA editor. Signal Averaged Electrocardiography Concepts, Methods and Applications. Lancaster: Kluwer Academic Publishers; 1993. p. 11-14. 